\title{
Control of Modular Mmmmultilevel Cascade Converters for offshore Wind Energy Generation and Transmission
}

\author{
Matias Diaz, Felix Rojas \\ Electrical Engineering Department \\ U. of Santiago of Chile \\ Santiago, Chile \\ matias.diazd@usach.cl
}

\author{
Felipe Donoso, Roberto Cardenas \\ Electrical Engineering Department \\ $U$. of Chile \\ Santiago, Chile \\ feldonoso@ing.uchile.cl,rcd@ieee.org
}

\author{
Mauricio Espinoza \\ School of Electrical Engineering \\ U. of Costa Rica \\ San Jose, Costa Rica \\ maeb@ieee.org
}

\author{
Andres Mora \\ Electrical Engineering Department \\ U. Santa Mara \\ Valparaiso, Chile \\ andres.mora@usm.cl
}

\author{
Patrick Wheeler \\ Power Electronics, Machines and Control Group \\ U. of Nottingham \\ Nottingham, United Kingdom \\ pat.wheeler@nottingham.ac.uk
}

\begin{abstract}
The trend of multimegawatt (Multi-MW) wind turbines, especially for offshore installations, has positioned Modular Multilevel Cascade Converters (MMCCs) as a promising solution for Wind Energy Conversion Systems (WECSs). Additionally, High Voltage Direct Current (HVDC) Transmission is likely to be the preferred option for future offshore wind farms. In both applications, Modular Multilevel Converters are an appropriate solution due to its characteristics such as highvoltage operation, controllability, redundancy, power quality and reliability. This paper presents the application of the Modular Multilevel Matrix Converter to locally interface a single wind turbine to a medium-voltage ac point of connection. Moreover, the Modular Multilevel Converter is proposed to link the wind farm to the grid onshore through an HVDC transmission line. Both converters are regulated using decoupled Vector Control Strategies. Experimental results obtained with a 27 Modular Multilevel Matrix Converter connected to a 30 power cell Backto-Back Modular Multilevel Converter are presented to validate the effectiveness of the Proposed Control Strategies.
\end{abstract}

\section{INTRODUCTION}

Among all renewable energy sources, wind energy has presented the most significant and fastest growth. The wind power production capacity for the whole world increased from 17.4 GW in 2000 to $486.8 \mathrm{GW}$ in 2016 [1]. A constant increase in wind power capacity is predictable in the immediate future. The European Wind Energy Association (EWEA) plan for the coming years is to make the wind industry the most competitive energy source, onshore by 2020 and offshore by 2030 [2].

Due to the presence of higher wind power potential and lower environmental impact, some of the future wind power

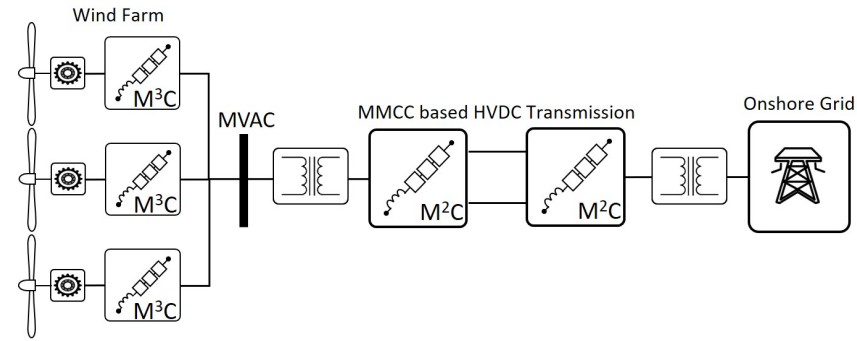

Fig. 1: MMCCs for offshore Wind Energy Generation and Transmission.

capacity will be installed offshore. For offshore applications, up-scaling wind turbine dimensions, wind farm capacities, and electrical infrastructure has become the focus of recent research. Large wind turbines can capture more power with less installations compared to a group of small wind turbines, and reduce the structure cost of offshore WECSs [3]. Owing to this fact, wind turbine nominal powers and rotor diameters have increased approximately to $10 \mathrm{MW}-160 \mathrm{~m}$ in 2015 [4]. The continuous increase in the power level of Wind Energy Conversion Systems (WECSs) is driving the power electronics technology towards MV operation. Accordingly, MV power converters will be dominant in the next generation MultiMW WECSs, mainly because they offer cost-effective and compact design [4], [5]. Modular Multilevel Cascade Converters (MMCCs) are an enabling technology which has been proposed recently for wind energy applications [6], [7], due to their several advantages over traditional topologies such as full modularity and easy extendability to reach high voltage 
levels, redundancy, control flexibility and power quality [8]

For the transmission of power from offshore WECSs, HVDC is gradually replacing High Voltage Alternant Current (HVAC) due to the reactive power generated by the cables using HVAC is considered as a limiting factor in longdistance transmissions lines. In HVDC transmission systems for wind farms, the converter topologies are either currentsource converters (CSCs) or voltage-source converters (VSCs). CSCs are used for power ratings over $100 \mathrm{MW}$, and it is mature technology regarding cost and reliability. For instance, line-commuted CSCs have been applied for offshore wind farms [9]. However, VSCs have been preferred over CSCs because of its capability to control active and reactive power independently, the possibility of connecting to weak grids and feasibility of operating multiterminal DC grids [10]. Recently, MMCC topologies has been proposed for HVDC wind farm [9]. Among the advantages of MMCCs over other VSC topologies for HVDC applications stand out [10] its modularity and scalability, MMCs can reach higher power ratings using standard semiconductors technology; its high efficiently and low distorted output voltages, as a consequence small filters are required to meet the grid codes.

In this context, this paper present an Vector Control Strategy for Wind Energy Generation and Transmission systems based on MMCCs. As shown in Fig. 1, this proposal considers the application of the Modular Multilevel Matrix Converter $\left(M^{3} C\right)$ to locally interface a single wind turbine to a mediumvoltage ac (MVAC) point of connection. Additionally, the Modular Multilevel Converter $\left(M^{2} C\right)$ is proposed to link the wind farm to the grid onshore through an HVDC transmission line. Both converters are controlled using an vector control system. This full based MMCCs configuration results in an high power density. For example, the MMCCs can be operated to synthesise frequencies to reduce the size of transformers and floating capacitors. Experimental results obtained with an 27 power cells $M^{3} C$ connected to a 30 power cell Back-toBack $M^{2} C$ are presented to validate the effectiveness of the proposed control strategy.

\section{Modular Multilevel Converters}

MMCCs are a relatively new family of power converters proposed initially for HVDC transmission [11]. The MMCC family is characterised by a cascade connection of power cells forming a cluster. These power cells are usually singlephase Full-Bridges or bidirectional choppers, and the converter voltage rating can be easily enlarged by increasing the number of cells per cluster. The capacitor voltage of each power cell is floating and could charge-discharge during the operation of the converter. Therefore, one of the most important control aims is to maintain the voltage in each capacitor within an acceptable range, particularly for variable-speed operation. Different connection of clusters and power cells, i.e. choppers or fullbridges, lead to different MMCCs topologies. A complete overview of the MMCC family is presented in [8].

The most well suited topologies for ac-to-ac applications are the $M^{2} C$ and the $M^{3} C$ [8]. The $M^{2} C$ performs dc-to-ac conversion and it comprises 6 clusters composed of chopper cells connected in series to an inductor. As shown in Fig. 2a, Back-to-Back $M^{2} C$ s connected by their dc ports are needed to allow bidirectional ac-dc-ac conversion. Fig. $2 \mathrm{~b}$ presents the normalised magnitude of the voltage oscillations in the floating capacitors as a function of the ac port frequency. Consequently, the control of the floating capacitors is difficult to perform when the ac system connected to its port is operating at zero or very low frequency [8], [12]. On the other hand, the $M^{3} C$ is composed of nine clusters based on the series connection of Full-Bridge power cells, as shown in Fig. 2c, which allows direct ac-to-ac connection of two ac ports. This converter is more suitable for low-speed high-power applications because lower circulating currents are required to mitigate the oscillations in the capacitors, in comparison to the $M^{2} C$ [13]. However, the $M^{3} C$ has an inherent problem when the input-port frequency is close to output port frequency (see Fig. 2b that presents the normalised capacitor voltage oscillations of the $M^{3} C$ as a function of the ratio of the inputoutput ports frequencies).

\section{A. MMCC for generation: $M^{3} C$}

The rotational speed of Multi-MW wind turbines is restricted within a range that depends on the wind speed, gearbox and generator [14]. In any case, the frequency of the Wind Turbine Input Port (e.g $20 \mathrm{~Hz}$ ) is lower than the frequency of the MVAC point of connection (e.g $50 \mathrm{~Hz}$ ) and low voltage oscillations appears in the floating capacitors (see Fig. 2b). Therefore, lower circulating currents are required to regulate the floating capacitors of the $M^{3} C$ resulting in a compact design of the converter [15]. In fact, the $M^{3} C$ is advantageously compared to others high-power converter topologies for low-speed applications [16], [17]. Therefore, it can be stated that the $M^{3} C$ is an appropriate solution to locally interface a Multi-MW wind turbine.

\section{B. MMCC for transmission: Back-to-Back $M^{2} C$}

On the contrary, low voltage oscillations appear in the floating capacitors of the $M^{2} C$ when the frequencies of its ports are not close to zero (see Fig. 2b) [8]. Therefore, lower circulating currents are required to regulate the floating capacitors of the $M^{2} C$ resulting in a compact design of the converter [12]. In fact, the $M^{2} C$ has been widely proposed for HVDC transmission [18]-[20].

\section{MMCCS MODELLING}

The dynamics of the $M^{3} C$ and B2B- $M^{2} C$ are represented by decoupled models obtained using linear transformations. The $M^{3} C$ can be represented by a decoupled model expressed in $\Sigma \Delta$ Double- $\alpha \beta 0$ coordinates as proposed in [15], [21], [22]. On the other hand, the Back-to-Back $M^{2} C$ can be represented by a decoupled model expressed in $\Sigma \Delta \alpha \beta 0$ coordinates as proposed in [12], [23].

In both cases, a Voltage-Current Model and a PowerCapacitor Voltage Model is obtained, and the use of the trans- 


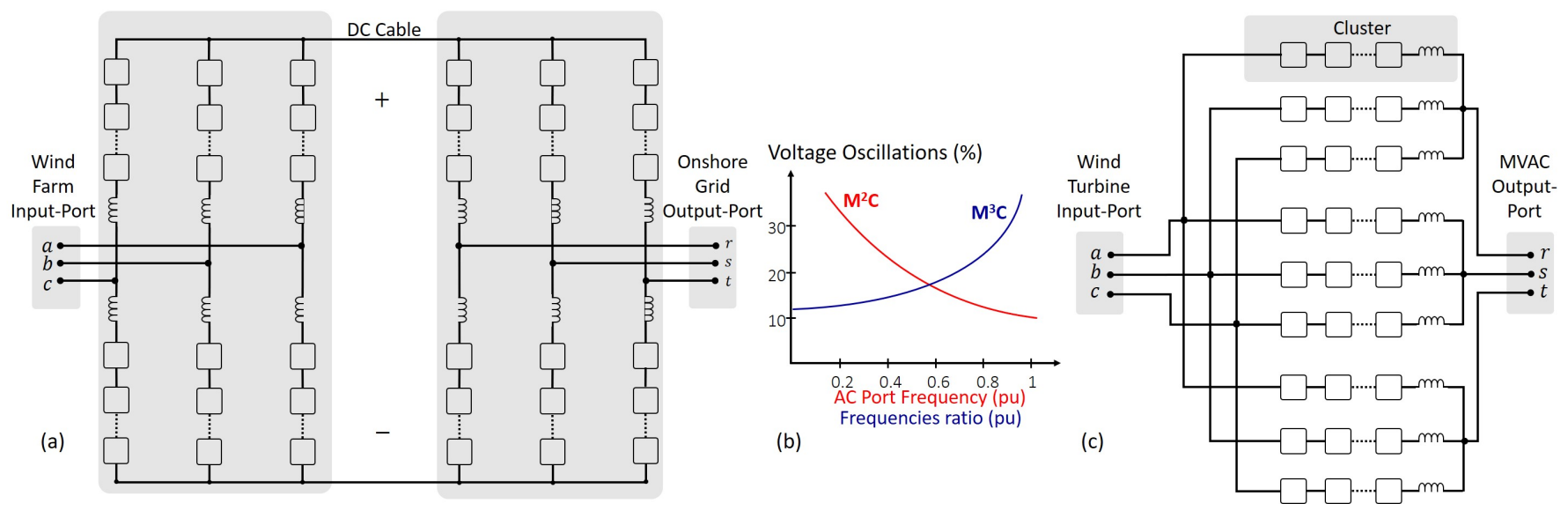

Fig. 2: (a) $M^{2} C$ circuit. (b) Normalised capacitor voltage oscillations. (c) $M^{2} C$ circuit.

formed cluster currents and voltages as degrees of freedom is enabled.

Furthermore, the sum of the $n$ capacitor voltages within a cluster is defined as the Cluster Capacitor Voltage (CCV) and it represents the available capacitor voltage. In both converters, there are two conditions to be satisfied to provide correct operation of the converters: the average component of the $\mathrm{CCV}$ must be equal in all the clusters in order to synthesise symmetrical voltages; and the capacitor voltages of the power cells within a cluster should be controlled to the same level. When both conditions are satisfied the $M^{3} C$ is balanced and the clusters can be considered as controlled voltage sources.

\section{A. Model of the $M^{3} C$}

\section{1) i) Voltage-Current Model of the $M^{3} C$ :}

The first model represents the currents and voltages of the $M^{3} C$. Applying Kirchhoffs Voltage Law to the $M^{3} C$ circuit the following expression is obtained:

$$
\begin{aligned}
& {\left[\begin{array}{lll}
v_{m a} & v_{m b} & v_{m c} \\
v_{m a} & v_{m b} & v_{m c} \\
v_{m a} & v_{m b} & v_{m c}
\end{array}\right]=L_{c} \frac{d}{d t}\left[\begin{array}{lll}
i_{a r} & i_{b r} & i_{c r} \\
i_{a s} & i_{b s} & i_{c s} \\
i_{a t} & i_{b t} & i_{c t}
\end{array}\right]+\left[\begin{array}{lll}
v_{a r} & v_{b r} & v_{c r} \\
v_{a s} & v_{b s} & v_{c s} \\
v_{a t} & v_{b t} & v_{c t}
\end{array}\right]} \\
& +\left[\begin{array}{lll}
v_{g r} & v_{g r} & v_{g r} \\
v_{g s} & v_{g s} & v_{g s} \\
v_{g t} & v_{g t} & v_{g t}
\end{array}\right]+v_{n}\left[\begin{array}{lll}
1 & 1 & 1 \\
1 & 1 & 1 \\
1 & 1 & 1
\end{array}\right]
\end{aligned}
$$

Where the subscript $m$ represents the input-port variables, and $g$ represents the output-port variables. $L_{c}$ is the cluster inductor inductance, and the common-mode voltage between the neutral points $N$ and $n$ is symbolised by $v_{n}$. Note that each array of capacitor in a cluster is replaced by a controlled voltage source $v_{x y}, x \in\{a, b, c\}, y \in\{r, s, t\}$.

In (1) the variables are coupled and the derivation of a control system is not straightforward. Then, the Double $\alpha \beta 0$ transformation [15] is used over (1) to obtain a decoupled model as follows:

$$
\begin{aligned}
& \sqrt{3}\left[\begin{array}{ccc}
0 & 0 & 0 \\
0 & 0 & 0 \\
v_{m_{\alpha}} & v_{m_{\beta}} & 0
\end{array}\right]=L_{c} \frac{d}{d t}\left[\begin{array}{ccc}
i_{\alpha \alpha} & i_{\beta \alpha} & i_{0 \alpha} \\
i_{\alpha \beta} & i_{\beta \beta} & i_{0 \beta} \\
i_{\alpha 0} & i_{\beta 0} & i_{00}
\end{array}\right] \\
& +\left[\begin{array}{lll}
v_{\alpha \alpha} & v_{\beta \alpha} & v_{0 \alpha} \\
v_{\alpha \beta} & v_{\beta \beta} & v_{0 \beta} \\
v_{\alpha 0} & v_{\beta 0} & v_{00}
\end{array}\right]+\sqrt{3}\left[\begin{array}{ccc}
0 & 0 & v_{g_{\alpha}} \\
0 & 0 & v_{g_{\beta}} \\
0 & 0 & 0
\end{array}\right]+\left[\begin{array}{ccc}
0 & 0 & 0 \\
0 & 0 & 0 \\
0 & 0 & 3 v_{n}
\end{array}\right]
\end{aligned}
$$

The currents $i_{\alpha 0}$ and $i_{\beta 0}$ are only dependent on the inputport currents, whereas $i_{0 \alpha}$ and $i_{0 \beta}$ are only dependent on the output-port currents. Furthermore, the currents $i_{\alpha \alpha}, i_{\beta \alpha}, i_{\beta \alpha}$ and $i_{\beta \beta}$ are known as Circulating Currents.

2) ii) Power-Cap. Voltage Model of the $M^{3} C$ :

The CCVs can be related to the cluster power as follows [15]:

$$
C v_{c}^{*} \frac{d}{d t}\left[\begin{array}{lll}
v_{c_{a r}} & v_{c_{a s}} & v_{c_{a t}} \\
v_{c_{b r}} & v_{c_{b s}} & v_{c_{b t}} \\
v_{c_{c r}} & v_{c_{c s}} & v_{c_{c t}}
\end{array}\right] \approx\left[\begin{array}{lll}
P_{a r} & P_{a s} & P_{a t} \\
P_{b r} & P_{b s} & P_{b t} \\
P_{c r} & P_{c s} & P_{c t}
\end{array}\right]
$$

Note that it is assumed that the power cells have the same capacitance $C$ and their capacitors are controlled to the desired value $v_{c}^{*}$. The Double $\alpha \beta 0$ Transformation is applied to (3) to enable a decoupled representation of the energy interaction among all the clusters of the $M^{3} C$ [22]. Accordingly, (3) results in:

$$
C v_{c}^{*} \frac{d}{d t}\left[\begin{array}{lll}
v_{c_{\alpha \alpha}} & v_{c_{\beta \alpha}} & v_{c_{0 \alpha}} \\
v_{c_{\alpha \beta}} & v_{c_{\beta \beta}} & v_{c_{0 \beta}} \\
v_{c_{\alpha 0}} & v_{c_{\beta 0}} & v_{c_{00}}
\end{array}\right] \approx\left[\begin{array}{ccc}
P_{\alpha \alpha} & P_{\beta \alpha} & P_{0 \alpha} \\
P_{\alpha \beta} & P_{\beta \beta} & P_{0 \beta} \\
P_{\alpha 0} & P_{\beta 0} & P_{00}
\end{array}\right]
$$

Thereafter, the $\Sigma \Delta$ Transformation is applied to (4) to improve the representation of the components $v_{c_{\alpha \alpha}}, v_{c_{\beta \alpha}}, v_{c_{\alpha \beta}}$ and $v_{c_{\beta \beta}}$ as a function of the input-output ports frequencies. Therefore, the Power-CCV of the $M^{3} C$ in $\Sigma \Delta \alpha \beta 0$ is obtained as follows:

$$
C v_{c}^{*} \frac{d}{d t}\left[\begin{array}{ccc}
v_{c_{1 \alpha}}^{\Sigma \Delta} & v_{c_{1 \beta}}^{\Sigma \Delta} & v_{c_{0 \alpha}} \\
v_{c_{2 \alpha}}^{\Sigma \Delta} & v_{c_{2 \beta}}^{\Sigma \Delta} & v_{c_{0 \beta}} \\
v_{c_{\alpha 0}} & v_{c_{\beta 0}} & v_{c_{00}}
\end{array}\right] \approx\left[\begin{array}{ccc}
P_{1 \alpha}^{\Sigma \Delta} & p_{1 \beta}^{\Sigma \Delta} & P_{0 \alpha} \\
P_{2 \alpha}^{\Sigma \Delta} & p_{2 \beta}^{\Sigma \Delta} & P_{0 \beta} \\
P_{\alpha 0} & P_{\beta 0} & P_{00}
\end{array}\right]
$$


The voltage terms of the left side of (5) represent voltage imbalances between different clusters. Additionally, $v_{c_{00}}$ is related to the total active power flowing into/from the $M^{3} C$.

\section{B. Model of the $M^{2} C$}

1) i) Voltage-Current Model of the $M^{2} C$ :

A decoupled model of the B2B- $M^{2} C$ is obtained considering only one side since both converters are symmetrical. Applying Kirchhoffs Voltage Law to the $M^{2} C$ circuit, the following expression is obtained:

$$
\begin{aligned}
L_{b} \frac{d}{d t}\left[\begin{array}{ccc}
i_{a}^{P} & i_{b}^{P} & i_{c}^{P} \\
i_{a}^{N} & i_{b}^{N} & i_{c}^{N}
\end{array}\right]=-\left[\begin{array}{ccc}
v_{a}^{P} & v_{b}^{P} & v_{c}^{P} \\
v_{a}^{N} & v_{b}^{N} & v_{c}^{N}
\end{array}\right] \\
+\left[\begin{array}{ccc}
-v_{a} & -v_{b} & -v_{c} \\
v_{a} & v_{b} & v_{c}
\end{array}\right]+\frac{E}{2}\left[\begin{array}{lll}
1 & 1 & 1 \\
1 & 1 & 1
\end{array}\right]
\end{aligned}
$$

Where the superscript $P$ represents the positive-pole cluster and $N$ represents the negative-pole cluster. The ac port variables are represented by $v_{x}$ and $i_{x}, x \in\{a, b, c\}, L_{b}$ is the cluster inductor inductance, and the dc port voltage is denoted by $E$. Note that each array of capacitor in a cluster is replaced by a controlled voltage source $v_{x}^{y}, y \in\{P, N\}$.

The $\Sigma \Delta \alpha \beta 0$ Transformation [12] is used over (6) to obtain a decoupled model as follows:

$L_{b} \frac{d}{d t}\left[\begin{array}{ccc}i_{\alpha}^{\Sigma} i_{\beta}^{\Sigma} & i^{P} \\ i_{\alpha} & i_{\beta} & 0\end{array}\right]=-\left[\begin{array}{ccc}v_{\alpha}^{\Sigma} & v_{\beta}^{\Sigma} & v_{0}^{\Sigma} \\ v_{\alpha}^{\Delta} & v_{\beta}^{\Delta} & v_{0}^{\Delta}\end{array}\right]-2\left[\begin{array}{ccc}0 & 0 & 0 \\ v_{\alpha} & v_{\beta} & v_{n_{0}}\end{array}\right]+\frac{E}{2}\left[\begin{array}{lll}0 & 0 & 1 \\ 0 & 0 & 0\end{array}\right]$

where the common-mode voltage is symbolised by $v_{n_{0}}$.

2) ii) Power-Cap. Voltage Model of the $M^{2} C$ :

The power of each cluster is estimated by Eq. (8) where $v_{C x}^{y}=\sum_{i=1}^{n} v_{C x_{i}}^{y}$ for $x \in\{a, b, c\}$ and $y \in\{P, N\}$, and the cluster power is calculated as $p_{x}^{y}=v_{x}^{y} i_{x}^{y}$.

$$
C v_{c}^{*} \frac{d}{d t}\left[\begin{array}{lll}
v_{C a}^{P} & v_{C b}^{P} & v_{C c}^{P} \\
v_{C a}^{N} & v_{C b}^{N} & v_{C c}^{N}
\end{array}\right] \approx\left[\begin{array}{lll}
p_{a}^{P} & p_{b}^{P} & p_{c}^{P} \\
p_{a}^{N} & p_{b}^{N} & p_{c}^{N}
\end{array}\right]
$$

Applying the $\Sigma \Delta \alpha \beta 0$ transformation to (8), the cluster power are defined by:

$$
C v_{c}^{*} \frac{d}{d t}\left[\begin{array}{lll}
v_{C \alpha}^{\Sigma} & v_{C \beta}^{\Sigma} & v_{C 0}^{\Sigma} \\
v_{C \alpha}^{\Delta} & v_{C \beta}^{\Delta} & v_{C 0}^{\Delta}
\end{array}\right] \approx\left[\begin{array}{lll}
p_{\alpha}^{\Sigma} & p_{\beta}^{\Sigma} & p_{0}^{\Sigma} \\
p_{\alpha}^{\Delta} & p_{\beta}^{\Delta} & p_{0}^{\Delta}
\end{array}\right]
$$

Where $p_{\alpha \beta}^{\Sigma}$ is related with the power flow between the converter phases; $p_{\alpha \beta 0}^{\Delta}$ represents the power flow between the upper and lower arm; and $p_{0}^{\Sigma}$ is related with the total energy of the converter.

\section{Vector Power-CCV Models}

The power components in the right-side of (4) and (9) can be expressed as a function of the transformed currents and voltages of the converters [12], [22].
1) i) Vector Power-CCV Model of the $M^{3} C$ :

Replacing the full expression of the power components of Appendix A into (4), and defining the power flows and CCVs of (5) as vectors, i.e. $\boldsymbol{P}_{1 \alpha \beta}^{\Sigma \Delta}=P_{1 \alpha}^{\Sigma \Delta}+j P_{1 \beta}^{\Sigma \Delta} \approx C v_{c}^{*} \frac{d}{d t} \boldsymbol{v}_{c_{1 \alpha \beta}}^{\Sigma \Delta}$, etc., the Power-CCV model of the $M^{3} C$ in $\Sigma \Delta$ Double- $\alpha \beta 0$ is obtained as follows:

$$
\begin{aligned}
C v_{c}^{*} \frac{d \boldsymbol{v}_{c_{\alpha \beta \beta}}^{\Sigma \Delta}}{d t} \approx & \frac{1}{6}\left(\boldsymbol{v}_{m_{\alpha \beta}}^{c} \boldsymbol{i}_{g_{\alpha \beta}}-\boldsymbol{v}_{g_{\alpha \beta}} \boldsymbol{i}_{m_{\alpha \beta}}^{c}\right) \\
& +\frac{1}{\sqrt{6}}\left(\boldsymbol{v}_{m_{\alpha \beta}} \boldsymbol{i}_{2 \alpha \beta}^{\Sigma \Delta}-\boldsymbol{v}_{g_{\alpha \beta}}^{c} \boldsymbol{i}_{2 \alpha \beta}^{\Sigma \Delta^{c}}\right)-v_{n} \boldsymbol{i}_{1 \alpha \beta}^{\Sigma \Delta} \\
C v_{c}^{*} \frac{d \boldsymbol{v}_{c_{2 \alpha \beta}}^{\Sigma \Delta}}{d t} \approx & \frac{1}{6}\left(\boldsymbol{v}_{m_{\alpha \beta}} \boldsymbol{i}_{g_{\alpha \beta}}-\boldsymbol{v}_{g_{\alpha \beta}} \boldsymbol{i}_{m_{\alpha \beta}}\right) \\
& +\frac{1}{\sqrt{6}}\left(\boldsymbol{v}_{m_{\alpha \beta}}^{c} \boldsymbol{i}_{1 \alpha \beta}^{\Sigma \Delta}-\boldsymbol{v}_{g_{\alpha \beta}}^{c} \boldsymbol{i}_{1 \alpha \beta}^{\Sigma \Delta^{c}}\right)-v_{n} \boldsymbol{i}_{2 \alpha \beta}^{\Sigma \Delta} \\
C v_{c}^{*} \frac{d \boldsymbol{v}_{c_{0}}^{\alpha \beta} \approx}{d t} \approx & \frac{1}{3 \sqrt{2}}\left(\boldsymbol{v}_{m_{\alpha \beta}}^{c} \boldsymbol{i}_{m_{\alpha \beta}}^{c}\right) \\
& -\frac{1}{\sqrt{3}}\left(\boldsymbol{v}_{g_{\alpha \beta}} \boldsymbol{i}_{1 \alpha \beta}^{\Sigma \Delta^{c}}+\boldsymbol{v}_{g_{\alpha \beta}}^{c} \boldsymbol{i}_{2 \alpha \beta}^{\Sigma \Delta}\right)-\frac{1}{\sqrt{3}} v_{n} \boldsymbol{i}_{m_{\alpha \beta}} \\
C v_{c}^{*} \frac{d \boldsymbol{v}_{c_{\alpha \beta}}^{0} \approx}{d t} \approx & \frac{-1}{3 \sqrt{2}}\left(\boldsymbol{v}_{g_{\alpha \beta}}^{c} \boldsymbol{i}_{g_{\alpha \beta}}^{c}\right) \\
& +\frac{1}{\sqrt{3}}\left(\boldsymbol{v}_{m_{\alpha \beta}} \boldsymbol{i}_{1 \alpha \beta}^{\Sigma \Delta}+\boldsymbol{v}_{m_{\alpha \beta}}^{c} \boldsymbol{i}_{2 \alpha \beta}^{\Sigma \Delta}\right)-\frac{1}{\sqrt{3}} v_{n} \boldsymbol{i}_{g_{\alpha \beta}}
\end{aligned}
$$

2) ii) Vector Power-CCV Model of the $M^{2} C$ :

The CCVs are defined by:

$$
\begin{aligned}
C v_{c}^{*} \frac{d \boldsymbol{v}_{C \alpha \beta}^{\Sigma}}{d t} & =-\frac{1}{4}\left(\boldsymbol{v}_{\alpha \beta} \boldsymbol{i}_{\alpha \beta}\right)^{c}+\frac{E}{2} \boldsymbol{i}_{\alpha \beta}^{\Sigma}-\frac{1}{2} v_{n_{0}} \boldsymbol{i}_{\alpha \beta} \\
C v_{c}^{*} \frac{d v_{C 0}^{\Sigma}}{d t} & =-\frac{1}{4} \Re\left\{\boldsymbol{v}_{\alpha \beta} \boldsymbol{i}_{\alpha \beta}^{c}\right\}+\frac{E i^{P}}{6} \\
C v_{c}^{*} \frac{d \boldsymbol{v}_{C \alpha \beta}^{\Delta}}{d t} & =-\left(\boldsymbol{v}_{\alpha \beta} \boldsymbol{i}_{\alpha \beta}^{\Sigma}\right)^{c}+\frac{E \boldsymbol{i}_{\alpha \beta}}{2}-\frac{2 i^{P} \boldsymbol{v}_{\alpha \beta}}{3}-2 v_{n_{0}} \boldsymbol{i}_{\alpha \beta}^{\Sigma} \\
C v_{c}^{*} \frac{d v_{C 0}^{\Delta}}{d t} & =-\Re\left\{\boldsymbol{v}_{\alpha \beta}\left(\boldsymbol{i}_{\alpha \beta}^{\Sigma}\right)^{c}\right\}-\frac{2}{3} i^{P} v_{n_{0}}
\end{aligned}
$$

\section{Proposed Vector Control Systems}

The overall control systems comprises an nested structure for decoupled regulation of the CCVs of each converter. As presented in Fig. 3, the input and the output port of each converter is independently controlled. In the following Subsections each control system is briefly described.

\section{A. Decoupled Vector Control $M^{3} C$}

The regulation of the floating capacitors of the $M^{3} C$ comprises the control of the component $v_{c_{00}}$ and the vectors $\boldsymbol{v}_{c_{1 \alpha \beta}}^{\Sigma \Delta}, \boldsymbol{v}_{c_{2 \alpha \beta}}^{\Sigma \Delta}, \boldsymbol{v}_{c_{0}}^{\alpha \beta}$, and $\boldsymbol{v}_{c_{\alpha \beta}}^{0}$. The average value of all the floating capacitor voltages is controlled using the component $v_{c_{00}}$, whereas the regulation of the four CCV vectors could be performed using either circulating currents or common-mode voltage $v_{n}$. In this proposal, the CCV vectors are controlled using circulating currents alone and $v_{n}=0$. 


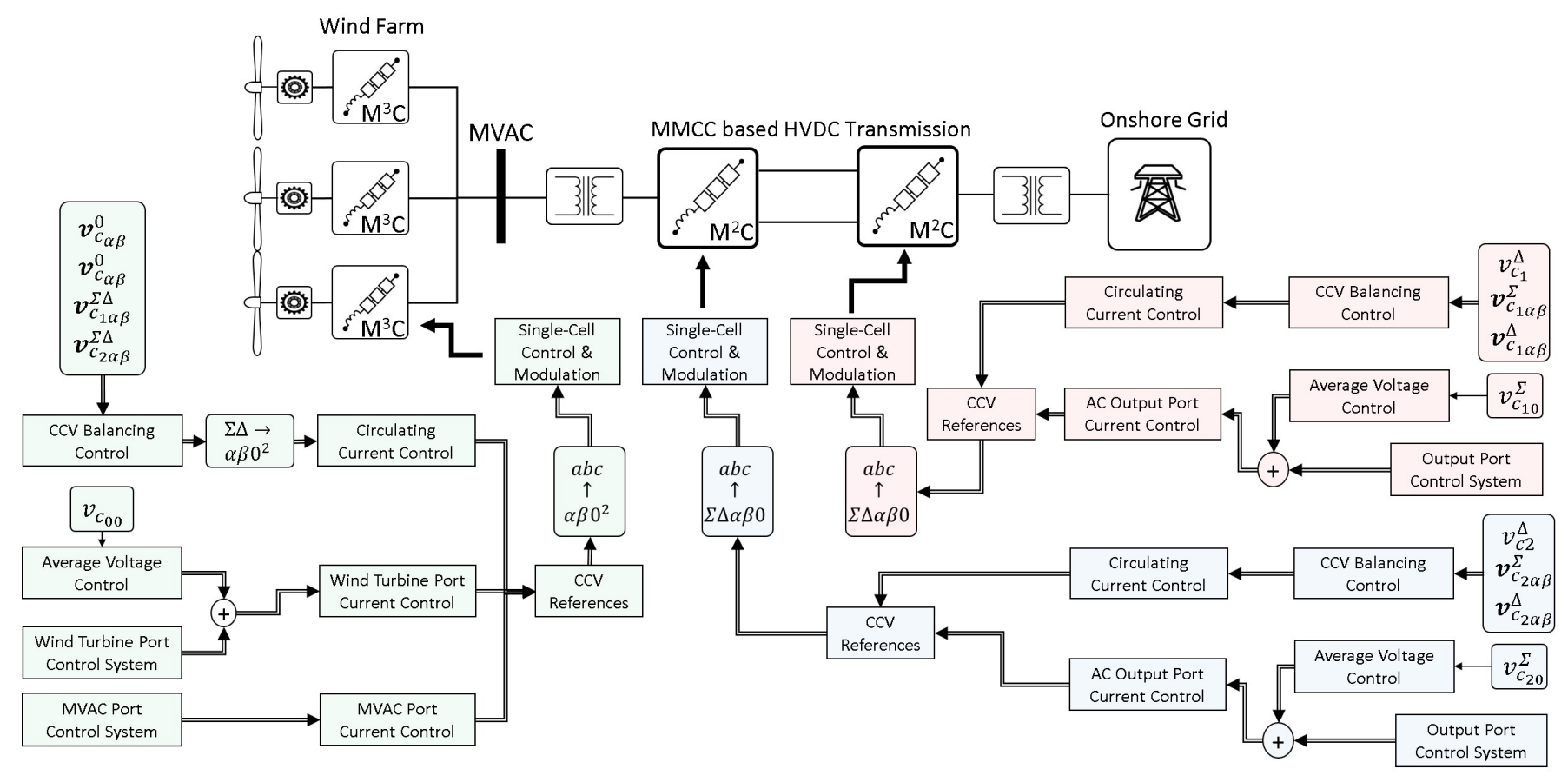

Fig. 3: Proposed Vector Control Strategy.

1) i) Control of $\boldsymbol{v}_{c_{1 \alpha \beta}}^{\Sigma \Delta}$ and $\boldsymbol{v}_{c_{2 \alpha \beta}}^{\Sigma \Delta}$ :

In $\Sigma \Delta \alpha \beta 0$, the vectors are defined as $\boldsymbol{x}_{\alpha \beta}^{\Sigma \Delta}=\boldsymbol{x}_{d q}{ }^{\Sigma \Delta} \mathrm{e}^{j \theta_{e}}$, where $\theta_{e}$ is the angle of vector. Then, (10)-(11) can be written as:

$$
\begin{aligned}
C v_{c}^{*} \frac{d \boldsymbol{v}_{c_{1 \alpha \beta}}^{\Sigma \Delta}}{d t} \approx & \frac{\left(\boldsymbol{v}_{m_{d q}} \boldsymbol{i}_{g_{d q}}-\boldsymbol{v}_{g_{d q}} \boldsymbol{i}_{m_{d q}}\right) \mathrm{e}^{j\left(\theta_{g}-\theta_{m}\right)}}{6} \\
& +\frac{\left(\boldsymbol{v}_{m_{d q}} \boldsymbol{i}_{2 d q}^{\Sigma \Delta}-\boldsymbol{v}_{g_{d q}} \boldsymbol{i}_{2 d q}^{\Sigma \Delta} \mathrm{e}^{-j\left(\theta_{g}-\theta_{m}\right)}\right)}{\sqrt{6}} \\
C v_{c}^{*} \frac{d \boldsymbol{v}_{c_{2 \alpha \beta}}^{\Sigma \Delta}}{d t} \approx & \frac{\left(\boldsymbol{v}_{m_{d q}} \boldsymbol{i}_{g_{d q}}-\boldsymbol{v}_{g_{d q}} \boldsymbol{i}_{m_{d q}}\right) \mathrm{e}^{j\left(\theta_{g}+\theta_{m}\right)}}{6} \\
& +\frac{1}{\sqrt{6}}\left(\boldsymbol{v}_{m_{d q}} \boldsymbol{i}_{1 d q}^{\Sigma \Delta}-\boldsymbol{v}_{g_{d q}} \boldsymbol{i}_{1 d q}^{\Sigma \Delta}\right) \mathrm{e}^{j\left(-\theta_{g}+\theta_{m}\right)}
\end{aligned}
$$

Considering that the rotational speed of the wind turbine connected to the input-port of the $M^{3} C$ is restricted within a suitable range, the first terms in the right-side of (18)-(19) possess components of frequencies $f_{m} \pm f_{g}$ that can be filtered by the capacitors [15].

Therefore, (18)-(19) yields to:

$$
C v_{c}^{*} \frac{d}{d t} \overline{\boldsymbol{v}}_{c_{1 \alpha \beta} \Delta}^{\Sigma \Delta} \approx \frac{\boldsymbol{v}_{m_{d q}} \boldsymbol{i}_{2 d q_{1}}^{\Sigma \Delta *}}{\sqrt{6}} ; v_{c}^{*} \frac{d}{d t} \overline{\boldsymbol{v}}_{c_{2 \alpha \beta} \Delta}^{\Sigma \Delta} \approx \frac{\boldsymbol{v}_{m_{d q}} \boldsymbol{i}_{1 d q_{1}}^{\Sigma \Delta *}}{\sqrt{6}}
$$

Note that (20) is valid when the circulating currents are defined as follows:

$$
\boldsymbol{i}_{1 \alpha \beta_{1}}^{\Sigma \Delta *}=\boldsymbol{i}_{1 d q_{1}}^{\Sigma \Delta *} \mathrm{e}^{j \theta_{m}} ; \boldsymbol{i}_{2 \alpha \beta_{1}}^{\Sigma \Delta *}=\boldsymbol{i}_{2 d q_{1}}^{\Sigma \Delta *} \mathrm{e}^{-j \theta_{m}}
$$

Hence, the active power flows produced by the right-side components of (20) are used to control the CCV vectors $\boldsymbol{v}_{c_{1 \alpha \beta} \Delta}^{\Sigma \Delta}$ and $\boldsymbol{v}_{c_{2 \alpha \beta}}^{\Sigma \Delta}$. The proposed balancing control system is depicted in Fig. 4. The outer control loop regulates with zero steady

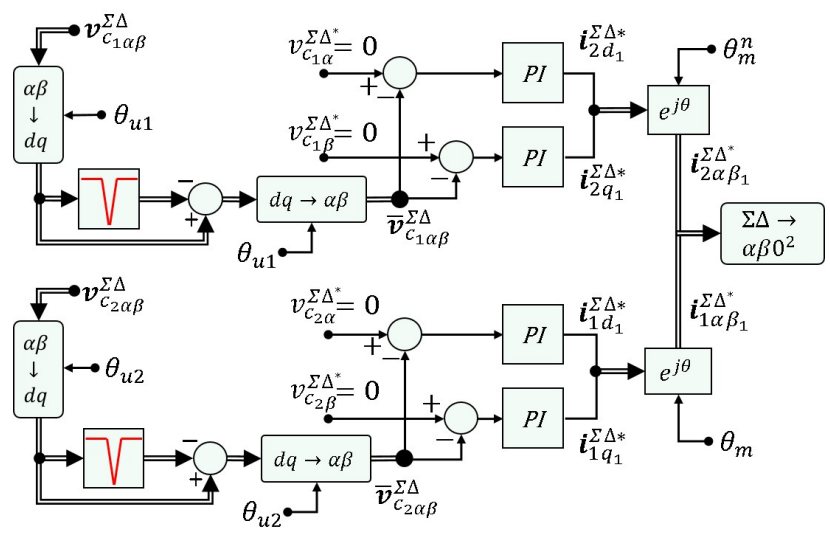

Fig. 4: Vector Control of $\boldsymbol{v}_{c_{1 \alpha \beta}}^{\Sigma \Delta}$ and $\boldsymbol{v}_{c_{2 \alpha \beta}}^{\Sigma \Delta}$

state error, using PI controllers, the average components of the vectors $\boldsymbol{v}_{c_{1 \alpha \beta}}^{\Sigma \Delta}$ and $\boldsymbol{v}_{c_{2 \alpha \beta}}^{\Sigma \Delta}$. The outputs of these external loops are used to calculate the reference of the $d q$ circulating currents.

2) ii) Control of $\boldsymbol{v}_{c_{0}}^{\alpha \beta}$ and $\boldsymbol{v}_{c_{\alpha \beta}}^{0}$ :

The same assumptions than in Section IV-A1 are valid for the regulation of $\boldsymbol{v}_{c_{0}}^{\alpha \beta}$ and $\boldsymbol{v}_{c_{\alpha \beta}}^{0}$, i.e. the first terms in the rightside of (12)-(13) possess components of frequencies $2 f_{m}$ and $2 f_{g}$ that can be filtered by the capacitors [15], the commonmode voltage is $v_{n}=0$ and the circulating currents are defined as:

$$
\boldsymbol{i}_{1 \alpha \beta_{2}}^{\Sigma \Delta *}=\boldsymbol{i}_{1 d q_{2}}^{\Sigma \Delta *} \mathrm{e}^{j \theta_{g}} ; \boldsymbol{i}_{2 \alpha \beta_{2}}^{\Sigma \Delta *}=\boldsymbol{i}_{2 d q_{2}}^{\Sigma \Delta *} \mathrm{e}^{j \theta_{m}}
$$

Then, dc components of the vector $\boldsymbol{v}_{c_{0}}^{\alpha \beta}$ are regulated to zero using a power flow in phase with the grid angle $\theta_{g}$. The same 
effect is achieved for $\boldsymbol{v}_{c_{\alpha \beta}}^{0}$ manipulating a non-zero mean active power in phase with the grid angle $\theta_{m}$ as follows:

$$
C v_{c}^{*} \frac{d}{d t} \overline{\boldsymbol{v}}_{c_{0}}^{\alpha \beta} \approx-\frac{\boldsymbol{v}_{g_{d q}} \boldsymbol{i}_{1 d q_{2}}^{\Sigma \Delta}}{\sqrt{3}} ; C v_{c}^{*} \frac{d}{d t} \overline{\boldsymbol{v}}_{c_{\alpha \beta}}^{0} \approx-\frac{\boldsymbol{v}_{m_{d q}} \boldsymbol{i}_{2 d q_{2}}^{\Sigma \Delta}}{\sqrt{3}}
$$

3) iii) Other Control Systems for the $M^{3} C$ :

a) Control of the average capacitor voltage component

The component $v_{c_{00}}$ represent the average voltage in all the capacitors of the $M^{3} C$ and it is related to the active power $P_{00}$ flowing into the converter. Therefore, the following expression can be written:

$$
P_{00}=P_{\text {in }}-P_{\text {out }} \approx C v_{c}^{*} \frac{d v_{c_{00}}}{d t}
$$

The term $P_{\text {out }}$ represents the $M^{3} C$ output power that is feedforwarded for control purposes. Therefore, the control system proposed in this work regulates the average voltage of all capacitors using a component of the input power. Readers can refer to [15], [21], [22] for more details.

\section{b) Circulating Currents Control}

The outputs of the Vector CCV control systems set the circulating current references in $\Sigma \Delta$ Double- $\alpha \beta 0$ coordinates. The circulating current references are transformed from $\Sigma \Delta$ Double- $\alpha \beta 0$ to $\alpha \beta 0^{2}$ coordinates and they are superposed yielding to the composite circulating current references. These references are regulated using proportional controllers. Readers can refer to [15], [21], [22] for more details.

\section{c) Input and Output Port Control Systems}

The model presented in (2) is decoupled and the currents $i_{\alpha 0}, i_{\beta 0}$ depend on the input-port currents, whereas $i_{0 \alpha}$ and $i_{0 \beta}$ depends on the output-port currents. Therefore, the inputport currents and the output-port currents can be controlled using conventional $d q$ control structures. Readers can refer to [15], [21], [22] for more details.

\section{d) Single-Cell Control and Modulation Scheme}

The voltage references obtained by the control systems presented in the previous are transformed to the natural reference frame using the inverse $\alpha \beta 0^{2}$ Transformation. Then, a CCV reference is obtained for each cluster in $a b c-r s t$ coordinates. Here, an additional control loop is utilised to regulate the capacitor voltages within a cluster at the same level [24].

\section{B. Decoupled Vector Control of the B2B- $M^{2} C$}

The control system of the B2B- $M^{2} C$ MMC is depicted in Fig. 5. Unlike $M^{2} C$-based drive converters [12], the $M^{2} C$ does not have considerable voltage oscillations in its flying capacitors for HVDC applications. As a consequence, it is not necessary to inject a common-mode voltage $v_{n_{0}}$ to balance the capacitor voltage modules. Moreover, the required circulating current is reduced. Since, in this case, the capacitor voltage oscillations are bounded in an acceptable region, the controllers only regulates the mean value of $\mathrm{CCVs}$ to its references.

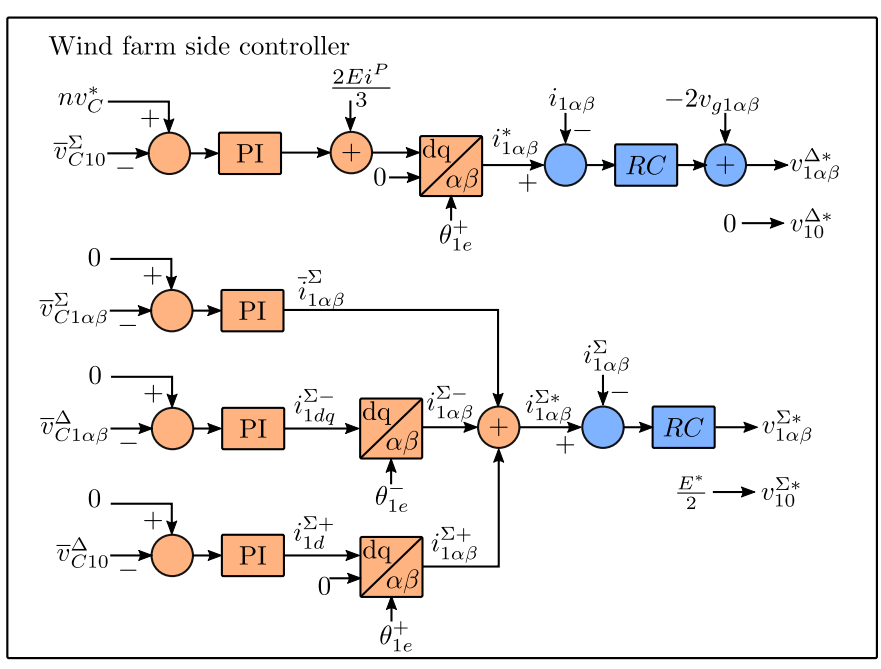

Fig. 5: Proposed Vector Control Strategy of the rectifier $M^{2} C$.

The proposed controller has a nested structure. An outer control loop balance the capacitor cluster voltages $v_{C \alpha \beta}^{\Sigma}$, $v_{C \alpha \beta}^{\Delta}, v_{C 0}^{\Delta}$ and $v_{C 0}^{\Sigma}$ for both sides, while an inner stage regulates the currents. The output of the outer loops gives the current references for $i_{\alpha \beta}$ and $i_{\alpha \beta}^{\Sigma}$. A notch filter is employed to obtain the dc component of $v_{C \alpha \beta 0}^{\Sigma \Delta}$. Each controller is explained below. The grid currents, circulating currents and grid voltages are defined as:

$$
\begin{aligned}
\boldsymbol{i}_{\alpha \beta} & =\boldsymbol{i}_{d q}^{+} \mathrm{e}^{\theta_{e}} \\
\boldsymbol{i}_{\alpha \beta}^{\Sigma} & =\overline{\boldsymbol{i}}_{\alpha \beta}^{\Sigma}+\boldsymbol{i}_{d q}^{\Sigma+} \mathrm{e}^{j \theta_{e}}+\boldsymbol{i}_{d q}^{\Sigma-} \mathrm{e}^{-j \theta_{e}} \\
\boldsymbol{v}_{\alpha \beta} & =v_{d} \mathrm{e}^{j \theta_{e}}
\end{aligned}
$$

\section{1) i) Control of $v_{C \alpha \beta}^{\Sigma}$ :}

By replacing $\boldsymbol{v}_{\alpha \beta}, \boldsymbol{i}_{\alpha \beta}$ and $\boldsymbol{i}_{\alpha \beta}^{\Sigma}$ from (25)-(27) into (14) and taking $v_{n_{0}}=0$ the power term $\boldsymbol{p}_{\alpha \beta}^{\Sigma}$ can be expressed as:

$$
\boldsymbol{p}_{\alpha \beta}^{\Sigma}=\frac{-\left(v_{d} \boldsymbol{i}_{d q}^{+}\right)^{c} \mathrm{e}^{-j 2 \theta_{e}}}{4}+\frac{E}{2}\left(\overline{\boldsymbol{i}}_{\alpha \beta}^{\Sigma}+\boldsymbol{i}_{d q}^{\Sigma+} \mathrm{e}^{j \theta_{e}}+\boldsymbol{i}_{d q}^{\Sigma-} \mathrm{e}^{-j \theta_{e}}\right)
$$

The power term $\boldsymbol{p}_{\alpha \beta}^{\Sigma}$ has one dc component and two ac components of frequency $f_{e}$ and $2 f_{e}$, however the ac components are filtered out by the $M^{2} C$ capacitors and only the $\frac{E}{2} \bar{i}_{\alpha \beta}^{\Sigma}$ produces a dc power term to regulate $\overline{\boldsymbol{v}}_{C \alpha \beta}^{\Sigma}$.

2) ii) Control of $v_{C \alpha \beta}^{\Delta}$

Analogously to the previous case, by replacing (25)-(27) into (15), the power term $p_{\alpha \beta}^{\Delta}$ can be calculated as:

$$
\begin{aligned}
\boldsymbol{p}_{\alpha \beta}^{\Delta}= & -\left(v_{d} \overline{\boldsymbol{i}}_{\alpha \beta}^{\Sigma}\right)^{c} \mathrm{e}^{-j \theta_{e}}-\left(v_{d} \boldsymbol{i}_{d q}^{\Sigma+}\right)^{c} \mathrm{e}^{-2 j \theta_{e}}-\left(v_{d} \boldsymbol{i}_{d q}^{\Sigma-}\right)^{c} \\
& +\left(\frac{E \boldsymbol{i}_{d q}^{+}}{2}-\frac{2 i^{P} v_{d}}{3}\right) \mathrm{e}^{j \theta_{e}}
\end{aligned}
$$

$\boldsymbol{p}_{\alpha \beta}^{\Delta}$ has components of frequency $0, f_{e}$ and $2 f_{e}$. Therefore, the current $i_{d q}^{\Sigma-}$ is employed to balance the voltage $\boldsymbol{v}_{C \alpha \beta}^{\Delta}$. A PI controller calculates $i_{d q}^{* \Sigma-}$. 


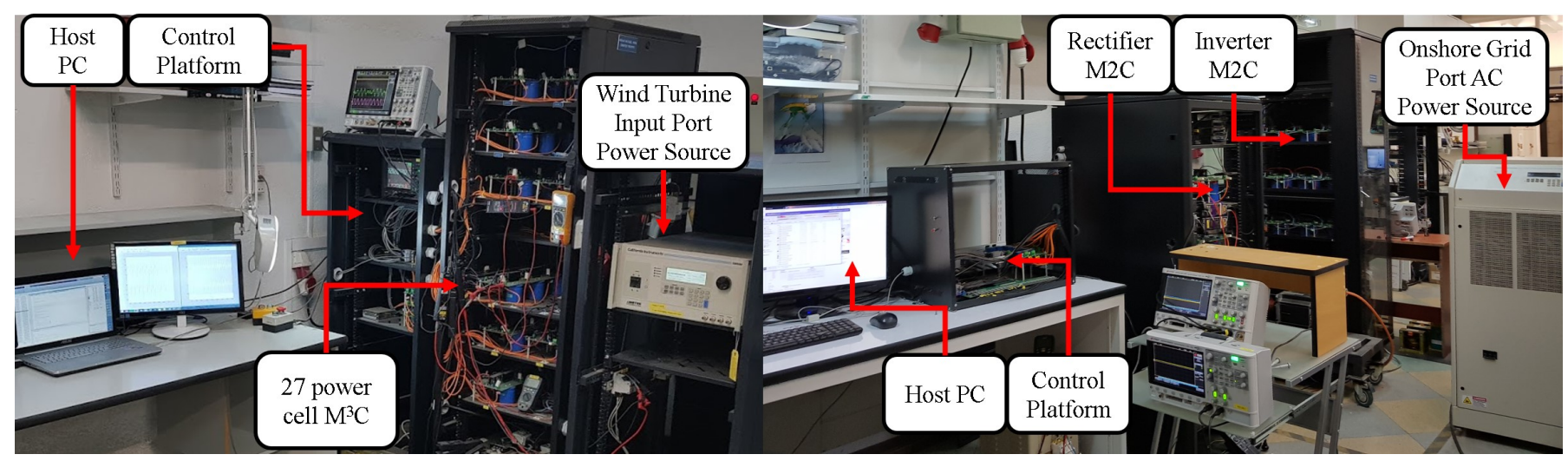

Fig. 6: Experimental Setup. Left-Side: $M^{3} C$. Right-side: B2B- $M^{2} C$.

3) iii) Control of $v_{C 0}^{\Sigma}$ and $v_{C 0}^{\Delta}$ :

Applying the same procedure the power terms $p_{0}^{\Sigma}$ and $p_{0}^{\Delta}$, can be calculated as (30)-(31) respectively. The proposed controller on Fig. 5 regulates $v_{C 0}^{\Sigma}$ using the direct current $i_{d}^{+}$. The wind farm side controller uses the feed-forward term $\frac{2 E i^{P}}{3}$ while the grid-side uses $\frac{2 P_{i} n}{3}$ to maintain the power balance between the AC ports of the HVDC system.

$$
\begin{aligned}
& p_{0}^{\Sigma}=-\frac{v_{d} i_{d}^{+}}{4}+\frac{E i^{P}}{6} \\
& p_{0}^{\Delta}=-\Re\left\{v_{d}\left(\boldsymbol{i}_{d q}^{\Sigma+}\right)^{c}+v_{d} \overline{\boldsymbol{i}}_{\alpha \beta}^{\Sigma} \mathrm{e}^{j \theta_{e}}+v_{d}\left(\boldsymbol{i}_{d q}^{\Sigma-}\right)^{c} \mathrm{e}^{j 2 \theta_{e}}\right\}
\end{aligned}
$$

4) iv) Other Control Systems for the $M^{2} C$ :

\section{a) Circulating Currents Control}

The voltage controllers of $v_{C \alpha \beta}^{\Sigma}, v_{C \alpha \beta}^{\Delta}$ and $v_{C 0}^{\Delta}$ calculates the current $i_{\alpha \beta}^{* \sigma}, i_{d q}^{* \sigma-}$ and $i_{d q}^{* \sigma+}$ respectively, these references are transform to the stationary $\alpha \beta$ frame. Resonant controllers are implement for the current inner loop $i_{\alpha \beta}^{\Sigma}$ and $i_{\alpha \beta}$ [12].

\section{b) Single-Cell Control and Modulation Scheme}

The controller outputs of the inner loops gives the voltage $v_{\alpha \beta 0}^{\Sigma \Delta}$. Using the inverse $\Sigma \Delta \alpha \beta 0$ transformation the cluster voltage reference $v_{a b c}^{P N}$ is obtained. This voltage is synthesized using phase-shifted modulation. As for the $M^{3} C$, an additional control regulates the individual capacitor voltage [21], [22]. The individual capacitor control regulates $v_{C}$ by charging/discharging its module and taking into account the cluster current sense.

\section{RESUlts}

Experimental results for the Proposed Vector Control System have been obtained using a 57-power cell $M^{3} C / \mathrm{B} 2 \mathrm{~B}-$ $M^{2} C$ prototype rated to $5 \mathrm{kVA}$. The power stage of the $M^{3} C$ prototype is composed of 27 Full-Bridge cells, whereas the power stage of the rectifier $M^{2} C$ is composed of 12 HalfBridge cells and the inverter $M^{2} C$ is composed of 18 HalfBridge cells. The $M^{3} C$, the rectifier $M^{2} C$ and the inverter $M^{2} C$ are operated using three control platforms comprised of a Texas Instrument DSP (TMS320C6713), FPGA boards
(Actel ProAsic3), external analogue-digital board, interface board with fibre optic transmitters and an HPI (Host Port Interface) daughter boards. The experimental setup is shown in Fig. 6 and the main parameters are provided in Table I. The dynamics of a single wind turbine is emulated using a programmable power source connected to Wind Turbine InputPort of the $M^{3} C$, which is then connected to the back to back $M^{2} C$. Finally, the Onshore Grid Output-Port of the B2B- $M^{2} C$ is connected to another Programmable Power Source.

\begin{tabular}{|c|c|}
\hline \hline Parameter & Value \\
\hline$M^{2} C$ Cell capacitor (both converters) & $2200 \mu \mathrm{F}$ \\
Output-Ports Voltages & $170 \mathrm{~V}$ \\
Output-Ports Frequencies & $50 \mathrm{~Hz}$ \\
Cluster inductor & $2.5 \mathrm{mH}$ \\
DC-Port Voltage $E$ & $450 \mathrm{~V}$ \\
\hline$M^{3} C$ Cell capacitor & $4700 \mu \mathrm{F}$ \\
Wind Turbine Port Voltages & $170 \mathrm{~V}$ \\
Wind Turbine Port Frequency & $16-40 \mathrm{~Hz}$ \\
MVAC Port Voltages & $200 \mathrm{~V}$ \\
MVAC Port Frequency & $50 \mathrm{~Hz}$ \\
Cluster inductor & $2.5 \mathrm{mH}$ \\
\hline \hline
\end{tabular}

TABLE I: Parameters of the experimental setup.

\section{A. Operation of the $M^{3} C$}

The operation of a variable-speed wind turbine is emulated using a wind speed profile from Rutherford Appleton Laboratories. As observed in Fig. 7(a), the wind speed profile generates a variable frequency at the Wind Turbine Input-Port of the $M^{3} C$, whereas the MVAC Output-Port is controlled to sinthetise $50 \mathrm{~Hz}$. The 27 floating capacitors are properly regulated to $v_{c}^{*}=150 \mathrm{~V}$ during the test regardless the InputPort frequency, as is shown in Fig. 7(b). The tracking of the direct and quadrature currents, presented in In Fig. 7(c), shows the the correct performance of the Input-Port Control System that obtain the maximum power point for each wind velocity regulating the quadrature current [25]. Finally, Fig. 7(d) presents the performance of the MVAC Output-Port of the $M^{3} C$, which is regulated to operate with unitary power factor to inject the active power produced by the wind turbine into the B2B- $M^{2} C$.

Oscilloscope waveforms are presented in Fig. 7e. From top 


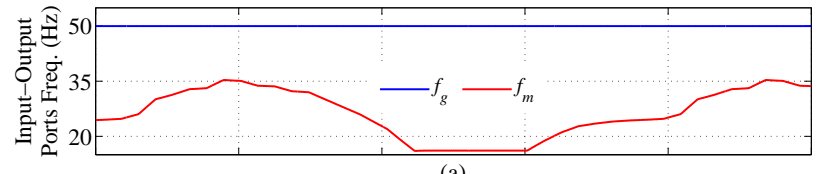

(a)

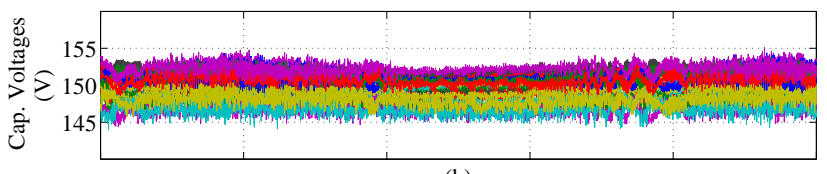

(b)
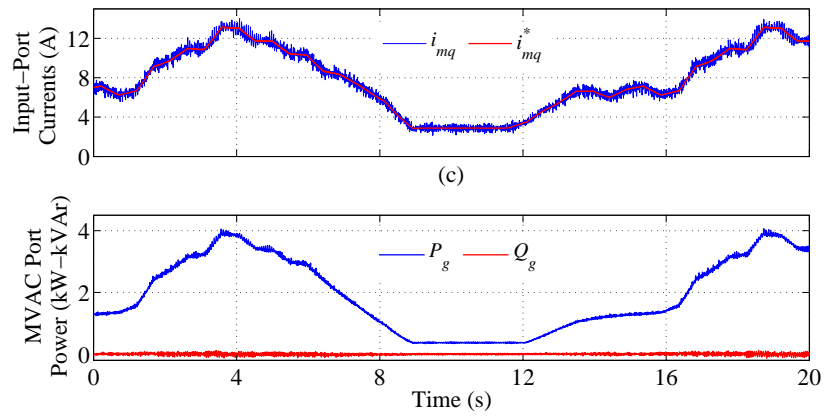

(d)
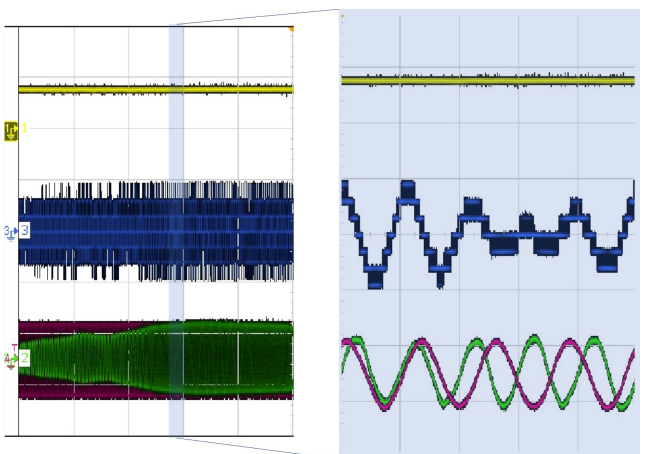

(e)

Fig. 7: Experimental Results for Variable-Speed Wind Turbine Emulation. (a) Grid and Generator Frequencies. (b) 27 Power Cells Capacitor Voltages. (c) Quadrature generator-side current tracking. (d) Active and Reactive Power Injected into the grid. (e) Oscilloscope Waveforms.

to bottom, one of the capacitor voltages $v_{c_{a r 1}}$, the cluster voltage $v_{a r}$, the grid voltage $v_{g_{r t}}$ (purple line) and the input voltage $v_{m_{a b}}$ (greem line), are presented. The cluster voltage $v_{a r}$ modulates both (Wind Turbine Input-Port and MVAC Output-Port) voltages and the different levels produced by the phase-shifted modulation are observed.

\section{B. Operation of grid-side $M^{2} C$}

Some relevant variables of the rectifier $M^{2} C$ are shown in Fig. 8. The system DC-port Current is $12 \mathrm{~A}$, whereas the DC-port Voltage is constant and its mean value is $\bar{E}=330 \mathrm{~V}$. The 12 floating capacitor of the rectifier $M^{2} C$ are regulated to $175 \mathrm{~V}$ and with low ripple. Fig. 8b shows the peak-topeak variance in the capacitors which is bounded in a $\pm 5 \mathrm{~V}$ band. Additionally, the circulating currents required for the regulation of the floating capacitors are low, as shown in Fig.
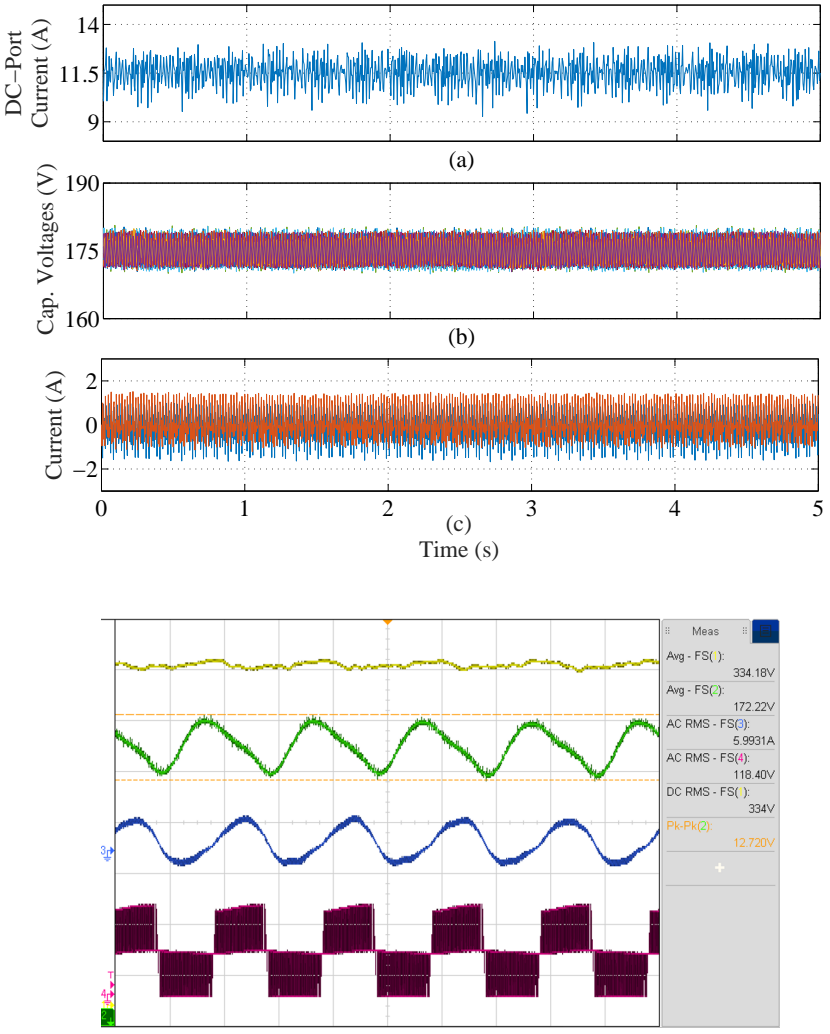

(d)

Fig. 8: Steady state response for the Rectifier $M^{2} C$. (a) DC-port Current. (b) 12 Power Cells Capacitor Voltages. (c) Circulating Currents. (d) Oscilloscope Waveforms.

8c. Oscilloscope waveforms are presented in Fig. 8d. From top to bottom, DC-port voltage $E$ (yellow), capacitor voltage $v_{C 1_{1}}^{P}$ (green), cluster current $i_{1 a}^{P}$ (blue) and cluster voltage $v_{a}^{P}$ (magenta) are depicted. The required circulating currents are reduced in comparison with the cluster current since the Wind Farm Input-Port frequency is $50 \mathrm{~Hz}$.

\section{CONCLUSION}

Vector Control Strategies based on the representation of the $M^{3} C$ in $\Sigma \Delta$ Double- $\alpha \beta 0$ coordinates and of the $M^{2} C$ in $\Sigma \Delta \alpha \beta 0$ coordinates have been proposed. The $M^{3} C$ is controlled to interface a single wind turbine to an MVAC point of connection. After that, the B2B- $M^{2} C$ is proposed to link several of this Multi-MW $M^{3} C$ based wind turbines to the grid through an HVDC transmission line. Both converters, the $M^{3} C$ and the $M^{2} C$ are controlled using decoupled vector control strategies.

The proposed Vector Control Strategies have been analytically discussed and their effectiveness has been validated through experiments conducted with a 57 power cell prototype. The experimental results have presented a correct performance for decoupled regulation of the Input-Output ports of the $M^{3} C$ and $\mathrm{B} 2 \mathrm{~B}-M^{2} C$. Additionally, the proposed Vector Control Strategies regulate the floating capacitors driving the voltage oscillations to zero. 


\section{ACKNOWLEDGMENT}

The support of University of Santiago of Chile through DICYT Projects $091813 D D$ and $091813 D D-R E D$ is kindly acknowledged.

\section{REFERENCES}

[1] Global Wind Energy Council, "Global Wind Statisitcs 2015. GWEC," Tech. Rep. 9, 2015.

[2] N. Fichaux, J. Beurskens, P. H. Jensen, and J. Wilkes, "Design limits and solutions for very large wind turbines: A 20 MW turbine is feasible," Tech. Rep. March, 2011.

[3] W. Erdman and M. Behnke, "Low Wind Speed Turbine Project Phase II : The Application of Medium-Voltage Electrical Apparatus to the Class of Variable Speed Multi-Megawatt Low Wind Speed Turbines Low Wind Speed Turbine Project Phase II : The Application of Medium-Voltage Electrical A," no. November, p. 119, 2005.

[4] M. Liserre, R. Cardenas, M. Molinas, J. Rodriguez, R. Cárdenas, M. Molinas, J. Rodríguez, R. Cardenas, M. Molinas, and J. Rodriguez, "Overview of Multi-MW Wind Turbines and Wind Parks," IEEE Trans. Ind. Electron., vol. 58, no. 4, pp. 1081-1095, apr 2011. [Online]. Available:

[5] V. Yaramasu, B. Wu, P. C. Sen, S. Kouro, and M. Narimani, "Highpower wind energy conversion systems: State-of-the-art and emerging technologies," Proc. IEEE, vol. 103, no. 5, pp. 740-788, 2015.

[6] R. Erickson, S. Angkititrakul, and K. Almazeedi, "A New Family of Multilevel Matrix Converters for Wind Power Applications: Final Report," University of Colorado, Tech. Rep. December, 2006.

[7] M. Díaz, R. Cárdenas, B. Mauricio Espinoza, A. Mora, and F. Rojas, "A novel LVRT control strategy for modular multilevel matrix converter based high-power wind energy conversion systems," in 2015 10th Int. Conf. Ecol. Veh. Renew. Energies, EVER 2015. IEEE, mar 2015, pp. $1-11$.

[8] H. Akagi, "Classification, terminology, and application of the modular multilevel cascade converter (MMCC)," IEEE Trans. Power Electron., vol. 26, no. 11, pp. 3119-3130, nov 2011.

[9] A. Egea-Alvarez, A. Junyent-Ferre, O. Gomis-Bellmunt, J. L. J. Ekanayake, and N. Jenkins, "Operation and control of VSC-HVDC multiterminal grids for offshore wind," EPE J. (European Power Electron. Drives Journal), vol. 23, no. 2, pp. 34-39, oct 2013.

[10] R. Vidal-Albalate, H. Beltran, A. Rolán, E. Belenguer, R. Peña, and R. Blasco-Gimenez, "Analysis of the Performance of MMC under Fault Conditions in HVDC-Based Offshore Wind Farms," IEEE Trans. Power Deliv., vol. 31, no. 2, pp. 839-847, apr 2016.

[11] A. Lesnicar and R. Marquardt, "A new modular voltage source inverter topology," in Proc. 10th Eur. Conf. Power Electron. Appl. EPE 2003, 2003.

[12] M. Espinoza, R. Cardenas, M. Diaz, and J. Clare, "An Enhanced dq-Based Vector Control System for Modular Multilevel Converters Feeding Variable Speed Drives," IEEE Trans. Ind. Electron., no. November, pp. 1-1, 2016.

[13] Y. Okazaki, W. Kawamura, M. Hagiwara, H. Akagi, T. Ishida, M. Tsukakoshi, and R. Nakamura, "Which is more suitable for MMCC-based medium-voltage motor drives, a DSCC inverter or a TSBC converter?" in 9th Int. Conf. Power Electron. - ECCE Asia "Green World with Power Electron. ICPE 2015-ECCE Asia. IEEE, jun 2015, pp. 1053-1060.

[14] V. Yaramasu and B. Wu, "Predictive control of a three-level boost converter and an NPC inverter for high-power PMSG-based medium voltage wind energy conversion systems," IEEE Trans. Power Electron., vol. 29 , no. 10 , pp. 5308-5322, oct 2014.

[15] M. Diaz, R. Cardenas, M. Espinoza, F. Rojas, A. Mora, J. C. Clare, and P. Wheeler, "Control of Wind Energy Conversion Systems Based on the Modular Multilevel Matrix Converter," IEEE Trans. Ind. Electron., vol. 64 , no. 11, pp. 1-1, nov 2017.

[16] L. Baruschka and A. Mertens, "A new three-phase AC/AC modular multilevel converter with six branches in hexagonal configuration," IEEE Trans. Ind. Appl., vol. 49, no. 3, pp. 1400-1410, may 2013.

[17] J. Kucka, D. Karwatzki, and A. Mertens, "AC/AC modular multilevel converters in wind energy applications: Design considerations," in 2016 18th Eur. Conf. Power Electron. Appl. (EPE'16 ECCE Eur. IEEE, sep 2016, pp. 1-10.
[18] A. Lesnicar and R. Marquardt, "An innovative modular multilevel converter topology suitable for a wide power range," in 2003 IEEE Bol. PowerTech - Conf. Proc., vol. 3. IEEE, 2003, pp. 272-277.

[19] R. Marquardt, "Modular Multilevel Converter: An universal concept for HVDC-Networks and extended DC-bus-applications," in 2010 Int. Power Electron. Conf. - ECCE Asia -, IPEC 2010. IEEE, jun 2010, pp. 502-507.

[20] M. Saeedifard and R. Iravani, "Dynamic performance of a modular multilevel back-to-back HVDC system," IEEE Trans. Power Deliv. vol. 25, no. 4, pp. 2903-2912, oct 2010.

[21] F. Kammerer, J. Kolb, and M. Braun, "Fully decoupled current control and energy balancing of the Modular Multilevel Matrix Converter," in 15th Int. Power Electron. Motion Control Conf. Expo. EPE-PEMC 2012 ECCE Eur. IEEE, sep 2012, pp. LS2a.3-1-LS2a.3-8.

[22] W. Kawamura, M. Hagiwara, and H. Akagi, "Control and experiment of a modular multilevel cascade converter based on triple-star bridge cells," IEEE Trans. Ind. Appl., vol. 50, no. 5, pp. 3536-3548, sep 2014. [Online]. Available:

[23] B. Mauricio Espinoza, A. Mora, M. Diaz, and R. Cárdenas, "Balancing energy and low frequency operation of the modular multilevel converter in back to back configuration," in 2015 10th Int. Conf. Ecol. Veh. Renew. Energies, EVER 2015. IEEE, mar 2015, pp. 1-9.

[24] H. Akagi, S. Inoue, and T. Yoshii, "Control and Performance of a Transformerless Cascade PWM STATCOM With Star Configuration," IEEE Trans. Ind. Appl., vol. 43, no. 4, pp. 1041-1049, 2007. [Online]. Available:

[25] M. Diaz, R. Cárdenas, M. Espinoza, A. Mora, and P. Wheeler, "Modelling and Control of the Modular Multilevel Matrix Converter and its application to Wind Energy Conversion Systems," in IECON 2016 - 42nd Annu. Conf. IEEE Ind. Electron. Soc., no. 1140337. IEEE, oct 2016, pp. 0-5. 